\title{
Trivium
}

Revue franco-allemande de sciences humaines et sociales - Deutsch-französische Zeitschrift für Geistesund Sozialwissenschaften

$13 \mid 2013$

Entre morale, politique et religion : la cohésion sociale selon Emile Durkheim

\section{Société, morale et individualisme. La théorie morale d'Emile Durkheim}

Hans-Peter Müller

Traducteur : Didier Renault

\section{OpenEdition}

Journals

Édition électronique

URL : https://journals.openedition.org/trivium/4490

DOI : $10.4000 /$ trivium.4490

ISSN : 1963-1820

Éditeur

Les éditions de la Maison des sciences de l'Homme

Référence électronique

Hans-Peter Müller, « Société, morale et individualisme. La théorie morale d’Emile Durkheim », Trivium

[En ligne], 13 | 2013, mis en ligne le 28 février 2013, consulté le 07 décembre 2022. URL : http://

journals.openedition.org/trivium/4490 ; DOI : https://doi.org/10.4000/trivium.4490

Ce document a été généré automatiquement le 29 septembre 2020.

Creative Commons - Attribution - Pas d'Utilisation Commerciale - Pas de Modification 4.0 International - CC BY-NC-ND 4.0

https://creativecommons.org/licenses/by-nc-nd/4.0/ 


\title{
Société, morale et individualisme. La théorie morale d'Emile Durkheim
}

\author{
Hans-Peter Müller
}

Traduction : Didier Renault

\section{NOTE DE L'ÉDITEUR}

Nous remercions M. Hans-Peter Müller ainsi que la maison d'édition Suhrkamp de nous avoir accordé l'autorisation de traduire ce texte pour le présent numéro.

Wir danken Herrn Hans-Peter Müller und dem Verlag Suhrkamp für die freundliche Genehmigung, diesen Artikel in französischer Übersetzung zu publizieren.

Un idéal n'est pas plus élevé parce qu'il est transcendant, mais parce qu'il nous offre de plus grandes perspectives [...] Nous ne sentons que trop à quel point il est difficile d'édifier cette société dans laquelle chaque individu aurait la place qu'il mérite, où chacun serait récompensé selon ses mérites et ou, par conséquent, tous concourent spontanément au bien-être de tous. ${ }^{1}$

\section{Introduction}

Peu de sociologues ont accordé autant d'importance à la morale dans leur système de pensée $e^{2}$ qu'Emile Durkheim (1858-1917). Dès le commencement de sa carrière scientifique, le fondateur de la sociologie universitaire française ${ }^{3}$ se livre à une confrontation critique avec la philosophie morale prédominante à son époque, de l'utilitarisme anglais de Jeremy Bentham, John Stuart Mill et Herbert Spencer à l'idéalisme allemand d'Emmanuel Kant, et conçoit le programme d'une science morale sociologique ${ }^{4}$. Ce programme met l'accent sur trois points et cherche des réponses aux 
questions suivantes : 1) A quoi devra ressembler une théorie morale prenant en compte la différenciation sociale et la division du travail des sociétés industrielles modernes? 2) Par quelles méthodes est-il possible d'analyser les phénomènes moraux ? 3) Quels sont les objectifs d'une morale individualiste progressiste, et par quels moyens peut-on l'aider à s'imposer dans tous les domaines de la vie sociale? Les accents théorique, méthodique et pratique convergent dans l'exigence suivante de Durkheim: "Notre premier devoir, aujourd'hui, consiste à fonder une nouvelle morale ${ }^{5} »$. Cette exigence est l'expression de sa profonde conscience d'une crise morale, puisqu'il attribue le malaise largement répandu dans les sociétés européennes à la rapide transformation d'une société traditionnelle organisée en corporations en une société industrielle. La conscience sociale n'a pas pu suivre le rythme des mutations multiples: révolution industrielle, triomphe d'un ordre économique capitaliste, révolution politique et émergence de conditions démocratiques, mais aussi révolution éthique et naissance d'un individualisme moral. L'incessante alternance de révolutions et de restaurations ${ }^{7}$ que connait la France du XIX ${ }^{e}$ siècle en est aux yeux du sociologue la meilleure preuve. Ce cours historique erratique de l'évolution sociale ne peut être rectifié, selon Durkheim, que si les deux grands foyers de crises de la Troisième République sont supprimés par une transformation sociale planifiée et par des réformes institutionnelles. Il s'agit d'une part de ce que l'on désigne comme la question sociale ${ }^{8}$ l'oppressante inégalité sociale dans l'économie doit être abolie en instituant la justice sociale dans les relations entre les entrepreneurs et les travailleurs; et d'autre part du système éducatif, où l'enjeu est de faire reculer l'influence conservatrice de l'Eglise catholique dans les écoles et de transmettre à la génération suivante, par l'édification d'un système laïque ${ }^{9}$, une conscience démocratique, la nécessaire solidarité sociale, et une morale individualiste. Ce n'est qu'ainsi que l'« unité organique ${ }^{10} »$ de la société sera renforcée et que l'édification d'une morale nouvelle pourra progresser.

2 En dépit de la conscience de la crise que nous avons notée et de l'usage largement synonyme, au XIX ${ }^{e}$ siècle, des notions de sciences sociales et de sciences morales ${ }^{11}$, la forte insistance de Durkheim sur les questions de morale paraissait déjà singulière à certains de ses contemporains ${ }^{12}$. Sa réception en Allemagne, par la suite, se heurta à des difficultés bien plus grandes, d'autant que le mot Moral, dans la langue allemande, a une signification très étroitement circonscrite, en partie limitée à la sphère privée, tandis que les termes français « morale » et «moralité » visent plutôt les valeurs, les normes et les règles sociales, la science morale étant par conséquent amenée à traiter de la structure et du développement de systèmes de valeurs. Peut-être faut-il attribuer à cette différence d'acception entre les deux langues le fait qu'on ait en Allemagne constamment reproché à Durkheim son sociologisme, son anti-individualisme et son conservatisme politique, et peut-être est-ce pour cette même raison qu'on a pu lire tout récemment une nouvelle invitation à se détourner de son paradigme ${ }^{13}$.

3 Avant de suivre cette recommandation, il nous faut néanmoins examiner si ces reproches sont réellement fondés ou s'ils ne reposent pas plutôt sur une réception simpliste. La présentation systématique de la théorie morale de Durkheim ${ }^{14}$ qui suit est destinée à montrer qu'il est impossible de maintenir ces reproches sous cette forme. Il est vrai que son approche collectiviste comporte une interprétation tout à fait particulière des idéaux sociaux et de l'autonomie personnelle, qui mène à une sorte d' individualisme collectiviste. Néanmoins, selon la thèse que je soutiens, Durkheim est en sociologie le théoricien d'une morale individualiste qui se préoccupe d'examiner les 
relations entre l'ordre social et la liberté individuelle ${ }^{15}$. Selon moi, il développe dans son programme de science morale trois arguments qui fondent la naissance, la préservation et l'idéalisation d'une morale individualiste. Il montre tout d'abord qu'une société pluraliste et fonctionnellement différenciée est structurellement contrainte d'offrir à ses membres des espaces de liberté pour l'action et l'organisation individuelle (argument de théorie structurelle). Il précise ensuite que la liberté individuelle n'est elle-même que le résultat socialisé d'un complexe processus d'éducation et de formation qui prépare aux plus divers droits et devoirs, et qui seul rend possible la conduite de la vie individuelle dans la société moderne (argument de théorie de la socialisation). Enfin, il démontre que l'unité morale, au sein de l'hétérogénéité des règles sociales, peut être atteinte par le nouvel idéal social, le culte religieux de l'individu, un idéal qui associe l'aspiration à l'autonomie personnelle et l'exigence de davantage de justice sociale (argument de sociologie religieuse).

Dans les pages qui suivent, nous développerons plus longuement ces trois arguments, en commençant par examiner les fondements du programme de sociologie morale de Durkheim, l'évolution historique des systèmes moraux, leurs conséquences sur l'éducation morale moderne et la solution proposée par le sociologue français pour résoudre la crise morale. A la lumière de ces réflexions, nous pourrons alors évaluer la validité de sa proposition, sa conception de "l'individualisme collectiviste ", et juger de manière critique la façon dont il détermine la relation entre ordre social et liberté individuelle.

\section{Le programme d'une science de la morale}

Dans ses deux premiers articles sur la philosophie et les sciences sociales en Allemagne ${ }^{16}$, qui donnent à Durkheim une subite notoriété en France et lui permettent d'obtenir un poste d'enseignant de sociologie à l'université de Bordeaux, s'exprime déjà clairement l'intention centrale de sa théorie morale. «En fait de toutes les philosophies qu'a produites l'Allemagne, le kantisme est celle qui, sagement interprétée, peut encore le mieux se concilier avec les exigences de la science ${ }^{17}$." Réaliser cette "sage interprétation " et transposer les acquis de l'idéalisme philosophique dans le cadre d'une théorie sociologique de la morale est l'idée directrice de la sociologie de Durkheim. Son programme, un kantisme sociologique, n'a pas pour objectif une métaphysique, mais une physique des mours et $d u d r o i t^{18}$, qui saisit, compare, classe les règles morales existantes - comme les valeurs, les mœurs, les usages et les conventions - et étudie leur influence sur la vie sociale.

6 Si Durkheim veut s'en tenir à la description que donne Kant des phénomènes moraux l'impératif catégorique, la liberté et l'autonomie de la volonté et le caractère obligatoire de la morale - il n'en refuse pas moins son explication philosophique. Selon lui, trois raisons expliquent pourquoi, jusqu'à présent, aucune théorie sociologique de la morale n'a pu naître de la philosophie morale idéaliste : la conception dualiste de la nature, la méthode déductive et l'universalité des doctrines morales ${ }^{19}$.

7 La sécularisation de la pensée inaugurée par les Lumières avait sans doute prouvé que l'organisation des sociétés n'était pas le résultat d'une volonté divine, mais celui de l'action humaine. A la suite de cette idée, on s'était cependant égaré dans l'illusion inverse, celle selon laquelle des organisations sociales peuvent être édifiées à tout moment et au gré d'une décision individuelle, l'être humain devant être considéré 
comme l'architecte exclusif de son organisation sociale. Toute tentative pour découvrir des lois de la vie sociale est alors vouée à être considérée par les gardiens de l'éthique comme une attaque contre le libre arbitre humain. Dans ces conditions, il est impossible de traiter scientifiquement des questions de l'ordre social et de la morale. Ce qui résulte au contraire de cet état de la pensée, c'est une conception dualiste de la nature, comme elle est propre au rationalisme cartésien, avec sa séparation entre l'esprit et la matière : la réalité se divise en un royaume des idées et des idéaux, uniquement accessible à la réflexion philosophique, et un royaume de la nature qui peut faire l'objet d'une étude scientifique. Selon Durkheim ${ }^{20}$, la seule possibilité pour s'extraire du corset de la conception dualiste est d'affirmer l'unité de la nature, en transposant l'idée du déterminisme aux organisations sociales et en s'efforçant de «traiter les faits de la vie morale selon les méthodes des sciences positives ».

Au postulat éthique du libre arbitre de l'être humain vient naturellement s'ajouter un argument supplémentaire, ontologique, qui découle de la conception dualiste de la nature et de la vision philosophique de la morale. Puisque les organisations sociales sont le résultat de conventions humaines, le résultat de ces conventions, la société, n'est elle-même qu'un artefact humain: "Une décision de la volonté les a faites, une autre décision peut les transformer ${ }^{21}$. » Si les sociétés sont des institutions entièrement artificielles, la philosophie morale doit s'appuyer sur l'unique véritable entité, la nature humaine. Telle est la raison plus profonde qui explique le procédé déductif à l'œuvre dans toutes les approches de la philosophie morale: on formule le principe moral suprême à partir d'une analyse de la nature humaine, et l'on déduit la totalité de la doctrine morale de ce principe suprême. Une telle déduction normative ne mène cependant qu'à un système conceptuel abstrait, postulé axiomatiquement et pourvu d'une logique interne, mais qui n'a aucun rapport avec la complexité et la diversité de la vie morale. Celle-ci ne peut être appréhendée que par l'induction empirique: «On ne peut pas construire la morale de toutes pièces pour l'imposer ensuite aux choses, mais il faut observer les choses pour en induire la morale ${ }^{22}$. »

9 Cette remarque vaut encore davantage pour l'universalité de la philosophie morale, car l'aspiration à une indépendance à l'égard de l'espace et du temps peut sans doute souligner la validité éthique de la doctrine, mais elle amoindrit toujours sa puissance explicative empirique. À titre d'exemple, si elle affirme que le principe moral suprême est la liberté, il devient impossible d'expliquer l'esclavage à partir des conditions sociales de la société antique, et l'on ne peut plus l'attribuer qu'au faible niveau éthique qu'avaient atteint les Romains. Au lieu d'une universalité de la philosophie morale, Durkheim part donc d'un relativisme de la sociologie morale, qui repose sur l'hypothèse

«que la morale de chaque peuple est directement en rapport avec la structure du peuple qui la pratique [...] chaque type social a la morale qui lui est nécessaire, comme chaque type biologique a le système nerveux qui lui permet de se maintenir. C'est donc que la morale est élaborée par la société même dont elle reflète ainsi fidèlement la structure ${ }^{23}$ ».

Seule cette hypothèse permet d'étudier les transformations historiques des conceptions morales dans leur dépendance vis-à-vis de l'évolution sociale et de vérifier s'il y a correspondance entre le type de société et le niveau moral. Là où existe cette correspondance règne l'équilibre, lorsqu'elle fait défaut, la société est plongée dans une crise morale, comme c'est le cas pour la France de l'époque, dans laquelle la conscience morale est en retard par rapport au niveau de l'évolution sociale. Le relativisme de la sociologie morale de Durkheim, qui permet de distinguer entre une évolution normale 
(équilibre) et pathologique (crise) n'affirme cependant pas que la morale moderne n'aurait aucune validité, ou seulement une validité « relative» : ce n'est le cas que lors d'une crise. Dans les périodes normales d'équilibre, elle déploie sa puissance impérative et a « pour fonction d'assurer l'ordre social ${ }^{24}$ ».

L'approche durkheimienne de la sociologie morale se fonde ainsi, du point de vue de la théorie de la connaissance, sur une conception moniste de la nature. Elle souligne l'unité des sciences naturelles et des sciences sociales, et sa méthodologie repose sur le procédé de l'induction empirique pour appréhender la diversité des phénomènes moraux dans leur genèse et leur mode de fonctionnement, et évaluer la normalité ou la déviance de leur évolution. Par conséquent, son programme d'une "physique des mœurs et du droit » ne vise pas à « tirer la morale de la science, mais [à] faire la science de la morale, ce qui est bien différent. Les faits moraux sont des phénomènes comme les autres; ils consistent en des règles d'action qui se reconnaissent à certains caractères distinctifs; il doit donc être possible de les observer, de les décrire, de les classer et de chercher les lois qui les expliquent ${ }^{25}$ ".

11 Mais à quoi reconnaît-on les règles morales, et quelles sont les caractéristiques qui les distinguent ? Si Durkheim ne souhaite pas exprimer d'emblée l'essence de la morale par une formule générale, il en donne une définition provisoire dans la Détermination du fait moral ${ }^{26}$, un texte qu'il propose en 1906 pour introduire une discussion à la Société française de philosophie. La communication, avec les interventions lors de la discussion qui lui ont été ajoutées, constitue le plus marquant et - avec le fragment sur la Morale et le cours sur l'Education morale ${ }^{27}$ - le plus abouti des textes de Durkheim sur les questions morales.

12 Partant de la distinction méthodologique selon laquelle on peut étudier la morale scientifiquement tout comme la juger dans la pratique, Durkheim choisit l'approche théorique et donne cette définition: "Toute morale se présente à nous comme un système de règles de conduite ${ }^{28}$. Ces règles « disent comment il faut agir dans des cas donnés; et bien agir, c'est bien obéir ${ }^{29}$ ». Mais agit-on moralement si, en République fédérale, on parle l'allemand, que l'on paie ses courses en marks et qu'en tant que chef d'entreprise, on "obéit ", dans une société de marché compétitive, à la loi de l'offre et de la demande ? Si la langue, la monnaie et les méthodes de production ${ }^{30}$ partagent avec les prescriptions morales une certaine contrainte sociale qu'elles exercent sur l'individu concerné, le type de " pression sociale » qui caractérise ces trois cas ne relève pas stricto sensu d'une norme morale, mais d'un ensemble de techniques et de moyens que l'on utilise pour parvenir à ses fins.

13 Afin de maintenir l'écart entre ces faits de nature diverse, Durkheim fait une distinction entre règles techniques et règles morales, et parvient ainsi à dégager la première caractéristique de la morale : l'obligation. Si celui qui enfreint des règles en vigueur s'expose toujours à des conséquences fâcheuses, la nature de ces conséquences diffère radicalement d'un type de règles à l'autre. Contrevenir à des règles techniques entraîne des conséquences négatives de manière immédiate et mécanique. Si l'on néglige, dans l'exemple que choisit Durkheim ${ }^{31}$, les règles élémentaires de l'hygiène, on tombe malade. En revanche, si l'on commet un homicide, on doit être sanctionné : cela signifie que l'on ne peut déduire de l'acte lui-même ses conséquences - en l'occurrence la nature et la gravité de la peine - mais que l'on doit prendre en compte la situation sociale (légitime défense ou assassinat) et la règle collective (la loi) concernant l'homicide. Tuer un ennemi à la guerre est une gloire et un honneur, alors qu'en temps 
de paix, c'est un odieux attentat contre l'intangibilité de la personne humaine. Dans le cas des règles techniques, l'action et ses conséquences sont reliées immédiatement et analytiquement; dans celui des règles morales, elles ne le sont que médiatement, par un lien synthétique, que Durkheim désigne sous le terme de sanctions. Le caractère d'obligation des règles morales apparaît en ce qu'elles énoncent des prescriptions et des interdits dont l'irrespect entraîne des sanctions sociales. Dans ce cas, la sanction « est une conséquence de l'acte qui ne résulte pas du contenu de l'acte, mais de ce que l'acte n'est pas conforme à une règle préétablie ${ }^{32}$ ». Les règles morales sont « investies d'une autorité spéciale en vertu de laquelle elles sont obéies parce qu'elles commandent ${ }^{33} »$.

Dans la mesure où il souligne le caractère d'obligation de la morale, Durkheim reste fidèle à l'analyse kantienne ; cependant il n'explique pas ce caractère d'un point de vue transcendantal - en partant de la nature humaine en laquelle résiderait cette conscience du devoir - mais d'un point de vue empirique et analytique, par le biais de l'examen des types de règles existantes et des effets qu'elles entraînent.

Toutefois l'obligation n'est que l'un des aspects de la morale, à laquelle doit s'ajouter un caractère de désirabilité. "Poursuivre une fin qui nous laisse froids, qui ne nous semble pas bonne, qui ne touche pas notre sensibilité, est chose psychologiquement impossible ${ }^{34}$. » Une pure éthique du devoir telle que la concevait Kant est aux yeux de Durkheim difficilement imaginable en pratique, et il a peine à comprendre pourquoi une personne devrait suivre une règle uniquement parce que cette dernière l'ordonne. Il manque ici le motif, l'intérêt propre, et même le plaisir de suivre la règle, qui ne peut avoir lieu que si l'individu « ressent » que l'objectif que poursuit la règle est bon.

Sur ce point, Durkheim reprend les idées de l'éthique utilitariste, selon lesquelles le respect de la règle est d'autant plus garanti que les intéressés veulent la règle, tandis que ce vouloir dépend lui-même de l'utilité que procure le mécanisme de la règle. Si un négociant fait de bonnes affaires grâce aux règles du marché, il sera un fervent partisan de l'économie de marché.

Toutefois, au-delà de cette compréhension pertinente du bien comme devant être voulu, les utilitaristes, qui soulignent excessivement l'élément hédoniste, oublient le caractère fondamental de l'action morale : le respect des règles, qui est lié à un certain effort, et même à un dépassement de soi, toujours inhérent au devoir.

La contrainte, le dépassement de soi et le devoir d'une part, de même que le sentiment altruiste, la désirabilité et la notion du bien de l'autre, sont deux propriétés centrales de la morale qui se complètent et s'interpénètrent dans la vision de Durkheim, et se confondent d'ailleurs dans les conduites individuelles observables. Ce que les idéalistes et les utilitaristes ont établi comme l'inconciliable opposition de principes moraux suprêmes s'intègre dans sa conception au titre d'éléments constitutifs et de rang égal de la moralité. Le devoir et le bien forment une unité non dépourvue de tensions, mais nullement contradictoire et ne sont à ses yeux que les deux faces de toute règle morale.

Durkheim précise cette relation étroite entre les deux éléments caractéristiques de la moralité, antagonistes seulement en apparence, par le concept du sacré, qui possède un caractère dualiste comparable. D'une part, le sacré inspire au croyant, sinon la crainte, du moins le respect ; d'autre part, il est un objet de désir, qui l'attire, le fascine, et dont il recherche le contact. Ces parallèles entre morale et religion ne sont pas pour Durkheim seulement le produit d'une analogie superficielle, mais l'expression d'une plus profonde similarité. Pendant des siècles, la morale a été de nature religieuse, 
religion et morale n'étaient pas encore séparées. Il est donc tout naturel que «la morale se présente à nous sous un double aspect : ici, comme une législation impérative et qui réclame de nous une entière obéissance, là, comme un magnifique idéal auquel la sensibilité aspire spontanément ${ }^{35} \%$.

Le devoir et le bien - ce qui signale déjà la parenté avec le sacré - sont deux éléments qui permettent, de manière tout à fait générale, de reconnaître les phénomènes moraux et qui valent aussi bien pour les systèmes moraux religieux que profanes. Mais qui incarne l'autorité impérieuse, qui est l'instance de sanction, qui remplace les dieux dans une morale séculière ? Ce ne peut être l'individu isolé, qui ne saurait inventer de lui-même, par l'introspection, une morale à validité collective - d'autant que les intérêts égoïstes n'ont jamais fondé une éthique. Il en va de même pour les intérêts particuliers d'un regroupement d'individus, de sorte qu'il ne reste plus que la société : " Donc, les fins morales sont celles qui ont pour objet une société. Agir moralement, c'est agir en vue d'un intérêt collectif ${ }^{36}$.»

«La société n'est donc pas, comme on l'a cru souvent, un événement étranger à la morale ou qui n'a sur elle que des répercussions secondaires ; c'en est, au contraire, la condition nécessaire. Elle n'est pas une simple juxtaposition d'individus qui apportent, en y entrant, une moralité intrinsèque; mais l'homme n'est un être moral que parce qu'il vit en société, puisque la moralité consiste à être solidaire d'un groupe et varie comme cette solidarité. Faites évanouir toute vie sociale, et la vie morale s'évanouit du même coup, n'ayant plus d'objet où se prendre ${ }^{37}$. "

21 Récapitulons: Toute morale se compose d'un système de règles de conduite qui inspirent le respect et sont en même temps attirantes et désirables. Sur la base de cette description générale, il est possible de poser la question de la naissance d'une morale individualiste, de son maintien par un complexe processus d'éducation et de socialisation, et de sa religiosité en tant qu'idéal collectif.

\section{Société, milieu moral et individu}

Dans De la division du travail social ${ }^{38}$, Durkheim étudie les conditions sociales nécessaires à l'émergence d'une morale individualiste. La division et la différenciation croissantes du travail ont fondamentalement transformé les règles morales de la coopération sociale. Il décrit cette transformation d'une morale collective et religieuse en une morale individualiste et séculière à travers les métamorphoses de la conscience collective et de la solidarité, autre manière de désigner le caractère moral d'une société. Afin de préciser la signification et la portée de cette transformation, Durkheim distingue deux idéaux-types de formes sociales: sociétés archaïques et sociétés modernes.

Les sociétés archaïques se composent de petites formations sociales que l'on peut aisément embrasser du regard et dont la vie en commun est strictement réglée par la tradition sacrée de normes et de pratiques religieuses. Le développement technique et économique, la division du travail, les communications et les transports sont peu évolués. Durkheim désigne ces sociétés tribales comme de type segmentaire parce que ces formations sociales homogènes sont dispersées sur un vaste territoire et entretiennent peu de liens entre elles. "Tant que chaque segment a sa vie qui lui est particulière, il forme une petite société dans la grande ${ }^{39}$." En raison de leurs dimensions modestes et de leur faible complexité, la vie de ces sociétés est réglée presque intégralement par la conscience collective de la communauté, c'est-à-dire par 
«l'ensemble des croyances et des sentiments communs à la moyenne des membres d'une même sociétée ${ }^{40}[. .$.$] ». Elle fait d'une part office de mémoire collective, en assurant$ la transmission des traditions et expériences du groupe d'une génération à l'autre ; d'autre part, elle fonde l'identité sociale, dans la mesure où elle intègre l'ensemble des règles morales qui garantit l'unité de l'expérience de la vie de tous les membres de la tribu et où elle maintient des conditions de vie identiques à travers les générations. Cette tradition extrêmement puissante est nécessairement de nature religieuse, d'autant plus que « la religion correspond à une région également très centrale de la conscience commune. [...] A l'origine, elle s'étend à tout; tout ce qui est social est religieux ; les deux mots sont synonymes ${ }^{41} »$.

Par conséquent, l'uniformité et la routine de la vie sociale suffisent à former l'individu selon les traits caractéristiques du collectif. Une éducation conçue comme un champ autonome et obligatoire n'y est pas nécessaire, puisque l'éducation dans l'ordre tribal a selon Durkheim pour principale caractéristique d'être "diffuse »: "Il n'y a pas de maîtres déterminés, pas de surveillants spéciaux préposés à la formation de la jeunesse ; c'est tous les anciens, c'est l'ensemble des générations antérieures qui joue ce rôle ${ }^{42}$.»

Durkheim caractérise ici l'intégration sociale de l'individu dans la collectivité comme une solidarité mécanique, qui, « née des ressemblances, rattache directement l'individu à la sociétét ${ }^{3}$ ». Plus la socialisation est parfaite, plus le degré de conformité sociale est élevé, et plus l'homogénéité entre la conscience morale de l'individu et celle de la société est forte. « La solidarité qui dérive des ressemblances est à son maximum quand la conscience collective recouvre exactement notre conscience totale et coïncide de tous points avec elle : mais, à ce moment, notre individualité est nulle ${ }^{44}$. »

Par opposition, la similarité des conditions d'existence et l'expérience de la vie partagée par la collectivité se désintègrent dans les sociétés évoluées. Les sociétés industrielles modernes sont de grandes nations caractérisées par un fort taux d'urbanisation, une disparité marquée entre la ville et la campagne, des systèmes de transports et de communications élaborés, un haut degré d'évolution technique, une économie des transports fortement développée avec une production pour le marché mondial. Cette nouvelle échelle, de même que la forte densité matérielle et morale comme Durkheim désigne également les voies de communication et de circulationfavorisent la division du travail, la différenciation sociale et la spécialisation fonctionnelle. On voit naître des sphères distinctes de la vie sociale ainsi que différents milieux sociaux qui ne peuvent plus être réglés par une conscience collective unique. Les nouvelles sphères de vie - tels que la grande industrie, l'Etat moderne et l'administration - doivent au contraire être soumises à des règles spécifiques et autonomes. "La division du travail donne naissance à des règles juridiques qui déterminent la nature et les rapports des fonctions divisées, mais dont la violation n'entraîne que des mesures réparatrices sans caractère expiatoire ${ }^{45}$. " Elle mène donc à une nouvelle forme de solidarité issue de la coopération, la solidarité organique, qui ne lie plus directement l'individu à la société dans son ensemble, mais aux sphères de vie et aux milieux dans lesquels il exerce son activité. En raison de la diversité des conditions de vie, l'individu ne peut plus être socialisé par une conscience collective, en tant que reproduction d'une mentalité commune unitaire. Au contraire, la solidarité organique impose rigoureusement la formation de personnalités autonomes, un processus dans lequel la liberté individuelle résulte de la participation à des contextes 
sociaux divers. C'est précisément son ancrage dans des milieux moraux différents qui prévient efficacement la subordination de l'individu à un groupe, parce qu'il lui faut se conformer à des obligations provenant de domaines distincts. Cela n'est possible qu'à une personnalité individuelle susceptible de coordonner les différentes exigences de comportement et de les harmoniser de manière sensée sur la base d'une conduite de vie autonome. Durkheim ramène donc "l'impératif catégorique de la conscience morale " à la formule : " Mets-toi en état de remplir utilement une fonction déterminée ${ }^{46}$. »

Une morale individualiste ne peut par conséquent fonctionner efficacement que si l'individu est soumis à un long processus d'éducation et de formation, seul apte à lui permettre une conduite de vie autonome. Une société industrielle différenciée par la division du travail ne pourra par conséquent développer une solidarité de coopération que si elle parvient à apporter à la génération suivante la nécessaire maîtrise de soi et le respect des règles - même en l'absence de la sanction de dieux courroucés -, à lui transmettre la connaissance du système de règles complexe d'une société moderne, avec ses participations à des groupes multiples, et à lui expliquer les perspectives et les risques d'une conduite de vie individuelle.

\section{L'éducation morale rationnelle dans une société séculière}

Dans L'Education morale ${ }^{47}$, Durkheim entreprend d'appliquer les connaissances issues de la sociologie morale à une éducation morale purement läque :

«par là, il faut entendre une éducation qui s'interdise tout emprunt aux principes sur lesquels reposent les religions révélées, qui s'appuie exclusivement sur des idées, des sentiments et des pratiques justiciables de la seule raison, en un mot une éducation purement rationaliste ${ }^{48}$ ".

Si le devoir et le bien sont des éléments constitutifs de toute morale, une morale individualiste qui ne repose sur aucune autre autorité que celle de la raison doit pouvoir présenter un élément supplémentaire, qui la distingue radicalement d'une morale fondée sur le sacré : une compréhension fondée, ou ce que Durkheim désigne volontiers comme la réflexion. Dans sa série de cours, il décrit trois processus de formation et de socialisation au cours desquels l'individu intériorise les valeurs, les règles et les normes morales : le sens du devoir doit être éveillé par l'esprit de discipline, car la maitrise de soi est la condition première de toute formation de la personnalité ; la désirabilité des idéaux sociaux doit résulter de l'attachement aux groupes sociaux, puisque l'intégration sociale de l'individu dans les différents groupes est la condition préalable de la solidarité organique; enfin la capacité de compréhension et de réflexion se forment grâce à l'autonomie, nécessaire pour permettre une conduite de vie individuelle. Esprit de discipline, attachement aux groupes sociaux et autonomie sont par conséquent les trois processus qui mènent à la personnalité dans le sens de l'humanisation, à la solidarité dans le sens de la sociabilité, et à l'individualité dans le sens de l'individuation. Ils constituent les caractéristiques spécifiques d'une morale individualiste.

\subsection{Discipline et développement de la personnalité humaine}

A première vue, il peut paraître étonnant que le thème du développement de l'autonomie morale soit introduit par une discussion sur la discipline. 
L'épanouissement personnel et la discipline morale ne s'excluent-t-ils pas mutuellement? L'individualité ne progresse-t-elle pas proportionnellement à la libération de la pression morale des réglementations et limitations sociales? Des utilitaristes tels que Hobbes et Bentham considèrent que la discipline est une violence faite à la nature individuelle, une violence néanmoins socialement nécessaire puisque les passions égoïstes ne pourraient sinon qu'aboutir au combat de tous contre tous : l'ordre social se paie ainsi au prix d'une limitation de la liberté individuelle.

Aux yeux de Durkheim, cette conception n'est pas seulement radicalement fausse parce qu'elle ne voit dans la discipline qu'un mal nécessaire au maintien de l'ordre, mais aussi parce qu'elle méconnaît le rôle positif que joue la discipline tout autant dans la coexistence sociale que dans le développement moral de la personnalité. Tout d'abord, la coopération sociale est impossible si le comportement individuel a lieu de manière totalement arbitraire et s'il est par conséquent foncièrement imprévisible. Le premier avantage de la discipline est qu'elle éveille le sens de la régularité et des habitudes de telle sorte que dans des circonstances identiques, on effectue les mêmes actions. Dans cette optique, la fonction de la morale est de déterminer la conduite par son autorité impérative, de la soumettre au contrôle social et de la soustraire à l'arbitraire individuel. Mais outre la préservation de l'ordre social, la discipline remplit deux autres fonctions qui participent de la socialisation de l'individu : « [elle] réalise[r] une certaine régularité dans la conduite des individus [et] leur assigne[r] des fins déterminées qui, du même coup, limitent leur horizon ${ }^{49}$." Lorsque cette régularité et ces buts font défaut, l'individu est livré sans défense à ses intérêts changeants. Un tel homme est-il autonome? Un despote tout puissant et qui ne connaît aucune limitation extérieure possède-t-il une liberté sans bornes? Durkheim estime qu'il devient très vite l'esclave de ses passions tyranniques, qu'il dégénère en jouet de ses penchants capricieux et que son omnipotence se transforme en une triste impuissance. La figure littéraire qui, au $\mathrm{XIX}^{\mathrm{e}}$ siècle, incarne le plus purement l'aspiration à l'infini est le Faust de Goethe. Aux yeux de Durkheim, Faust est moralement incomplet parce qu'il ne s'impose aucune limitation extérieure, parce qu'il ne peut se décider pour une science déterminée et concentrer sur elle toutes ses forces, parce qu'il poursuit des buts qui sont par définition impossibles à atteindre. " La maîtrise de soi, voilà la première condition de tout pouvoir vrai, de toute liberté digne de ce nom ", de telle sorte qu'agir moralement "c'est apprendre aussi à se conduire avec suite, d'après des principes constants, supérieurs aux impulsions et aux suggestions fortuites. C'est donc communément à l'école du devoir que se forme la volonté ${ }^{50}$ ».

31 L'idée d'une limitation raisonnable et d'un équilibre moral dans la vie individuelle et sociale exprime une idée cardinale de la sociologie morale de Durkheim. Comme l'a déjà montré la Division du travail, c'est le destin de l'homme moderne d'occuper une fonction déterminée et d'être un élément d'un ensemble plus vaste. Dans tous les cas où l'homme tente de transcender ces limitations et de ne pas tenir compte de sa propre nature - comme il le démontre dans Le Suicide ${ }^{51}-$, l'équilibre est rompu entre ses capacités et ses possibilités, entre ses buts et ses moyens, et il est en proie à une grave crise. L'impulsion débridée à s'élever met en péril inconsidérément le bonheur de l'individu ; car Durkheim est convaincu que c'est une dangereuse illusion de croire que le bonheur croîtrait « sans limites, avec le pouvoir, le savoir, la richesse ${ }^{52}$ ».

Résumons : 
«La discipline est donc utile, non pas seulement dans l'intérêt de la société, et comme le moyen indispensable sans lequel il ne saurait y avoir de coopération régulière, mais dans l'intérêt même de l'individu. C'est par elle que nous apprenons cette modération du désir sans laquelle l'homme ne saurait être heureux. Et, par là, elle contribue même, pour une large part, à former ce qu'il y a de plus essentiel en chacun de nous, je veux dire notre personnalité53.»

\subsection{Attachement au groupe social et pluralité de l'appartenance à des groupes dans les sociétés différenciées}

33 Avec l'esprit de discipline et la maitrise de soi comme fondement de tout agir moral, on a désigné un premier processus de formation et d'éducation qui ne peut être mis en œuvre sans l'attachement au groupe social. L'être humain est sans aucun doute un être social, et il a besoin de l'atmosphère morale d'une collectivité au même titre que d'oxygène pour respirer. Dans la conception de Durkheim, si la société est l'instance suprême de l'autorité morale, le processus de socialisation place toujours l'individu en interaction avec plusieurs groupes sociaux. Seul l'individu archaïque est directement intégré à la collectivité tribale, tandis que l'individu moderne est membre d'une famille, d'une école, d'un groupe professionnel, d'une société et de l'humanité tout entière. Ces milieux sociaux distincts possèdent des règles dont la portée et la généralité varient et se situent donc à des niveaux d'ordre différents. Puisqu'une règle est d'autant plus générale qu'elle est impersonnelle, la famille, la profession, l'Etat et l'humanité forment selon Durkheim une hiérarchie. Au niveau le moins élevé, la famille veille à la socialisation élémentaire de l'enfant, offre la chaleur humaine et les sentiments moraux qui permettent l'attachement au premier groupe social. S'appuyant sur sa loi de contraction ${ }^{54}$, Durkheim signale néanmoins la perte de fonction qualitative et quantitative de la famille nucléaire moderne, qui n'a pas seulement dû abandonner à d'autres sphères sociales un certains nombre de tâches économiques, politiques et juridiques, mais se heurte également à une forte concurrence dans la fonction de socialisation qui lui reste. L'éducation publique, et avant tout l'école, inaugure la seconde socialisation, la deuxième période de l'enfance, qui prépare l'enfant à la vie dans une société où règne la division du travail.

Si l'impératif catégorique de la conscience morale moderne consiste en l'exercice d'un métier, la morale professionnelle prend une importance croissante à titre d'importante médiation entre morale familiale et morale citoyenne. Tandis que dans la sphère publique - l'Etat, l'administration et l'armée - les groupements professionnels sont déjà largement répandus, ils font totalement défaut dans l'industrie et le commerce, depuis que toutes les corporations intermédiaires ont été abolies pendant la Révolution française. Face à l'anomie régnant sur l'économie et à la " question sociale ", Durkheim propose ${ }^{55}$ de constituer des groupes professionnels comme organes de réglementation autonomes, pour régler les conflits entre employeurs et employés par la conciliation de leurs intérêts respectifs.

Ce sont cependant l'Etat et l'humanité qui constituent le degré le plus général et le plus élevé de la morale, et Durkheim se pose la question de savoir lequel, du nationalisme ou du cosmopolitisme, devrait être l'idéal suprême d'une morale citoyenne. Ce qui plaide contre le cosmopolitisme est qu'il n'existe pas de société mondiale qui pourrait garantir la préservation de l'idéal, même si dans l'idéal de la fraternité humaine et dans le culte de l'individu, le représentant de l'espèce et le type individuel se confondent. Malgré la 
possibilité d'une société européenne, « l'Etat est actuellement le groupe humain le plus organisé qui existe ${ }^{56} »$, et Durkheim défend par conséquent un patriotisme réfléchi, tourné vers l'intérieur, auquel devrait se conformer la nation française. Il n'entend pas par là un nationalisme ni un impérialisme tournés vers l'extérieur, mais la réalisation d'idéaux cosmopolites au plan de l'organisation politique, de telle sorte que la nation française aspire au degré le plus élevé possible de justice sociale et à la meilleure organisation morale, et qu'elle devienne ainsi un modèle pour tous les autres Etats.

C'est dans la mesure où l'individu s'intègre à la société au cours du processus de socialisation, qu'il s'incorpore aux différents groupes sociaux et qu'il intériorise la hiérarchie des droits et des devoirs moraux qu'il considérera les idéaux impersonnels comme désirables et qu'il sera en mesure de porter la responsabilité d'une solidarité issue de la coopération. Toutefois, à ce stade, est-il déjà autonome pour autant?

\subsection{Autonomie personnelle et individualisme moral}

Comme nous l'avons déjà vu grâce aux deux premiers processus, l'autonomie ne doit pas être confondue avec un épanouissement personnel sans limitations, une réalisation de soi autarcique et détachée de la société. Au contraire, la discipline est le fondement essentiel de la maîtrise de soi, la condition indispensable de notre aptitude à la moralité, tandis que l'attachement aux groupes sociaux est le processus par lequel nous nous approprions au fur et à mesure les acquis civilisateurs de la société, faisant ainsi progresser la construction de notre personnalité.

A y regarder de plus près, les deux premières composantes du processus de socialisation et d'éducation - acquisition d'une discipline et intériorisation de la hiérarchie des normes - sont des processus parfaitement hétéronomes et difficiles à concilier avec l'exigence d'autonomie. Comment réconcilier le caractère externe et imposé des règles morales, qui pour devenir imperceptible dans le cours du développement de la personnalité n'en demeure pas moins réel, avec l'exigence d'autonomie personnelle de la conscience morale moderne? Comment une morale rationnelle peut-elle prendre en compte l'exigence d'autodétermination selon laquelle on ne peut qualifier de morale une règle que lorsqu'elle a été établie sans contrainte, en toute liberté et en tant qu'acte de libre adhésion? En bref, comment est-il possible de concilier le déterminisme moral et le libre arbitre?

Durkheim explique tout d'abord la possibilité de l'autonomie personnelle à travers la relation entre l'homme et la nature. Au fur et à mesure des progrès des sciences de la nature, qui révèlent toujours plus de lois du monde physique, le sentiment que les hommes maitrisent celui-ci ne cesse de se renforcer. Nous ne nous sentons plus esclaves, mais maîtres de la nature, bien que nous n'ayons pas créé les lois de l'univers, et qu'en dépit de tout notre savoir, nous y restions soumis dans une large mesure. La compréhension du " plan de la nature » que nous permet la science suffit déjà à nous donner ce sentiment de liberté.

Selon Durkheim, il n'en va pas autrement de la vie morale. « Comme la morale exprime la nature de la société, et que celle-ci n'est pas plus connue directement de nous que la nature physique, la raison de l'individu ne peut pas plus être la législatrice du monde moral que celle du monde matériel ${ }^{57}$. Ce constat ne signe pourtant pas nécessairement une capitulation de la raison humaine. Au contraire, plus la science morale est évoluée et plus les lois morales 
de l'ordre social ressortent nettement, plus on comprend leur sens et leur but. Cette représentation cognitive des règles dans la conscience du sujet lui ouvre déjà " un premier degré d'autonomie ${ }^{58} »$. En outre, la raison exige un examen critique et une analyse qui évalue une règle morale du point de vue de sa conformité aux principes d'une morale individualiste. Ces processus de connaissance et de jugement favorisent la compréhension de la nécessité de la morale sociale : dès lors, le libre consentement au monde moral n'est pas le signe d'une soumission mais un acte volontaire de l'autonomie personnelle.

«Et par là-même, nous redevenons nos maîtres. C'est la pensée qui est libératrice de la volonté. Cette proposition, que tout le monde admettra volontiers pour ce qui concerne le monde physique, n'est pas moins vraie dans le monde moral ${ }^{59}$. 》

L'argumentation rationaliste de Durkheim soulève trois questions au sujet de la relation entre morale sociale et morale individuelle. Que se passe-t-il lorsque l'examen critique aboutit non pas à un consentement volontaire, mais à un refus fondé ? Dans quels cas la conscience morale individuelle est-elle en droit de se rebeller contre la morale en vigueur? Et pourquoi des personnalités moralement et intellectuellement mûres ne sont-elles pas capables d'élaborer leurs propres principes moraux ?

La meilleure solution pour résoudre cet ensemble de problèmes est d'en revenir à la conception dualiste de la nature humaine ${ }^{60}$ de Durkheim. Il opère en effet une distinction entre le corps, ou organisme, comme siège de la sensibilité, avec l'ensemble des passions, des pulsions et des penchants individuels d'une part, et l'esprit, ou personnalité, le siège de l'intellection, avec toutes les connaissances sociales, les règles et les sentiments moraux de l'autre. Il ressort nécessairement de cette opposition entre être individuel et être social que la personnalité est un produit de la société: «La morale individuelle [...] est même au plus haut point sociale. Car ce qu'elle nous prescrit de réaliser, c'est le type idéal de l'homme tel que le conçoit la société considérée ; or un idéal, chaque société le conçoit à son image ${ }^{61}$. Selon cette conception, nul ne peut, de manière générale, refuser la morale sociale en tous points, puisque l'instance d'évaluation, la personnalité, n'est elle-même qu'une copie du type collectif de l'individu. Si Durkheim concède tout de même l'existence d'une morale individuelle parce qu'il y a toujours des "fautes de copie ", que le cercle de la socialisation n'est jamais totalement clos et que chacun se fait une image quelque peu différente de la morale sociale - ces variations n'ouvrent cependant pas une grande latitude à l'initiative morale personnelle. Elles signifient seulement que «chacun de nous est immoral par certains côtés ${ }^{62}$ » en raison d'une socialisation incomplète.

Par conséquent, la personnalité ne peut avoir de motif pour refuser à titre individuel des règles existantes que lorsque la morale en vigueur reste en retard sur les possibilités qu'ouvre l'évolution structurelle de la société. Dans ce cas, la rébellion individuelle ne signifie cependant pas un refus de la société, mais la tentative de la faire évoluer. En ce sens, Durkheim décrit les innovations morales de Socrate et de Jésus comme la tentative de personnalités géniales de formuler l'idéal déjà présent dans la structure sociale et de donner à voir à leurs contemporains l'image de l'évolution future. Mais il serait faux de penser que Socrate et Jésus auraient pu élaborer leur doctrine morale hors du temps et de l'espace et détachés des conditions sociales spécifiques. Durkheim n'a donc pas une très haute opinion des projets moraux utopiques totalement incompatibles avec l'état actuel de la société, puisqu'ils lui rappellent l'illusion du primitif «qui, par un acte de sa volonté, par un désir exprimé, 
par une injonction énergique, croit pouvoir arrêter la marche du soleil, contenir la tempête ou déchaîner les vents ${ }^{63}$ ».

La tentative de l'homme moderne d'élaborer de manière autonome les principes d'une conduite de vie individuelle n'est cependant pas aussi irréaliste et absurde que la pratique magique du primitif. En effet, l'argument de théorie structurelle de Durkheim renvoie à l'hétérogénéisation des règles morales et à la contrainte qui pousse à l'individuation, tandis qu'il s'efforce, dans son argumentation en termes de théorie de la socialisation, de fixer à nouveau des bornes morales aux espaces de liberté qui viennent de naître et de les soustraire à la transformation individuelle par une règlementation collectiv(ist)e. Cette tension entre arguments de théorie structurelle et de la socialisation provient de la mise en parallèle exagérée, qui risque d'étouffer l'élément de l'autonomie, entre morale archaïque et morale moderne. Si la morale archaïque comporte dans tous les cas un conformisme obligatoire ${ }^{64}$ qui repose sur une relation asymétrique entre le collectif survalorisé religieusement et l'individu croyant, l'aspiration à l'autonomie de la morale séculière exige que les individus eux-mêmes fixent les règles de leur coopération d'égal à égal, ce qui fonde une relation symétrique entre le collectif et l'individu. Dans les deux cas, les individus contractent des obligations sociales; mais tandis qu'elles proviennent dans le premier d'une morale contraignante imposée, elles découlent dans le second d'une morale librement consentie de la coopération. En outre, l'autonomie morale signifie qu'en cas de conflit entre une règle morale universaliste et une norme sociale, la personne se conforme au principe universaliste, transcendant ainsi le motif social que Durkheim a en vue.

La difficulté à déterminer la relation entre morale sociale et morale individuelle provient en dernier ressort du parti-pris unilatéralement sociologique de Durkheim, qui consiste à penser la morale tout comme la personnalité individuelle à partir de la perspective de la société. C'est aussi ce que montre le caractère très sommaire de sa théorie de la personnalité, puisque si Durkheim décrit bien le dualisme de la nature humaine dans son champ de forces, la perspective d'une méta-instance susceptible d'intégrer au sein de l'individu les forces divergentes de l'organisme et de la personnalité de manière à permettre une conduite de vie raisonnable fait défaut. Il manque à son système une conception comparable à la théorie des instances de Freud ${ }^{65}$, dans laquelle le Moi rétablit l'équilibre entre les exigences concurrentes du ça et du surmoi, ou un théorème de l'identité comme celui de "l'identité pour soi» de Goffman $^{66}$ qui permet d'accorder l'identité personnelle et l'identité sociale. Il apparaît sur cet arrière-plan que sa détermination de la relation entre ordre social et liberté individuelle repose sur un individualisme collectiviste qui s'adresse à un type individuel schématique ${ }^{67}$, et ne met pas l'accent sur le caractère irremplaçable et unique d'une biographie humaine, comme s'efforcent de le faire de plus récentes théories du sujet et de l'identité.

La mise en parallèle de la morale archaïque et de la morale moderne par le biais de l'analogie avec le sacré mène finalement Durkheim hors du cercle étroit des processus de formation et d'éducation morale, jusqu'à un problème relevant de la sociologie religieuse : celui de savoir comment le contenu de la conscience collective moderne, l'individualisme moral, pourrait devenir un idéal collectif «sacré » et une moderne religion de la citoyenneté. 


\section{La crise morale, l'affaire Dreyfus et le culte de l'individu} continue d'exister après le processus de sécularisation et soulève la question de savoir comment s'incarnent, dans une morale rationnelle marquée par l'individualisme, ces fondements sacrés et par quels processus ils acquièrent une efficacité fonctionnelle. Fondamentalement, on retrouve dans cette interrogation le vieux problème des Lumières françaises, celui de comprendre comment les progrès rationnels de la pensée pouvaient être associés au socle de croyances d'une société sécularisée. Rousseau ${ }^{68}$ proposait dans ce but d'instituer une religion citoyenne, autrement dit une religion civile, qui réalise l'intégration de l'ordre social sans enfreindre les droits de la raison.

Pendant toute sa vie, Durkheim s'est exclusivement penché sur les systèmes religieux archaïques, sans jamais transposer les connaissances ainsi acquises aux sociétés modernes, à une exception près : l'article L'Individualisme et les intellectuels ${ }^{69}$, sa prise de position de principe sur l'Affaire Dreyfus. C'est pourquoi cet article, qui révèle une fois encore que la sociologie de Durkheim est une science de crise, est instructif du point de vue historique et systématique : il éclaire les arrière-plans de la plus grave crise de la Troisième République et met en évidence, par le fossé qui sépare les dreyfusards des antidreyfusards, les profondes divisions de la société française. En même temps, il présente l'image que se fait Durkheim d'une société bien ordonnée ${ }^{70}$, de telle sorte qu'avec les réflexions qui concluent l'article sur l'individualisme moral comme religion séculière, nous sommes en mesure de compléter sa vision de la conciliation de l'ordre social et de la liberté individuelle.

Peu de temps après la condamnation du capitaine Dreyfus, l'enjeu de la discussion publique n'est déjà plus seulement la légitimité du jugement, mais les reproches des antidreyfusards selon lequel les intellectuels, avec leur individualisme égoïste et athée, sapent la raison d'Etat et mènent la société française à l'anarchie morale et spirituelle. Durkheim $^{71}$ repousse résolument cette accusation en opérant une distinction entre individualisme utilitariste et individualisme moral. L'utilitarisme célèbre en effet un " culte égoïste du moi », ne connaît qu'un dogme, celui de «l'apothéose du bien-être et de l'intérêt privé", et un seul rite, la "doctrine de l'utile». Il faut en revanche distinguer radicalement de tout cela l'individualisme moral, avec son «culte de l'individu », son unique dogme, "l'autonomie de la raison », et son rite, « la doctrine du libre examen ». Là où la première conception aboutit effectivement à l'anarchie morale, la seconde mène à la solidarité organique et à l'ordre social. Pourtant, comment parvenir à cet ordre si toutes les opinions individuelles restent libres et que les règles ne sont acceptées que par un accord volontaire?

En premier lieu, l'individualisme moral est effectivement inconcevable sans un certain degré d'intellectualisme, parce qu'une morale rationnelle se fonde nécessairement sur la liberté de pensée. Cependant, cette liberté ne signifie pas que l'individu ferait de sa pensée et de ses actes le critère unique de toutes choses. Au contraire : de même que le scientifique emprunte couramment sans les vérifier des savoirs issus d'autres domaines, l'individu se plie à l'avis d'autrui pour peu qu'il lui présente de bonnes raisons pour cela. Ces bonnes raisons instituent elles-mêmes la nécessaire autorité scientifique et morale qui est au fondement du principe cardinal de la solidarité organique, la réciprocité sur la base de l'égalité. De plus, la coopération sociale produit les idées et 
sentiments communs nécessaires, qui se sont concentrés, dans les sociétés modernes, dans le culte de l'individu. L'individualisme moral est l'expression du caractère sacré de la personne :

«Cette personne humaine [...] est considérée comme sacrée, au sens rituel du mot pour ainsi dire. Elle a quelque chose de cette majesté transcendante que les Eglises de tous les temps prêtent à leurs Dieux [...] Et c'est précisément de là que vient le respect dont elle est l'objet. [...] Une telle morale n'est donc pas simplement une discipline hygiénique ou une sage économie de l'existence ; c'est une religion dont l'homme est, à la fois, le fidèle et le Dieu ${ }^{72}$."

51 Cet aspect religieux explique pourquoi la conscience publique se montre aussi sensible au traitement juridique douteux qu'a subi le capitaine Dreyfus. L'événement est perçu comme un sacrilège à l'encontre de l'idéal collectif sacré, celui de la liberté et de l'intangibilité de la personne humaine. L'indignation publique n'est par conséquent pas seulement provoquée par la pitié pour la victime, mais vise également à la purification et à la restauration de l'idéal religieux. La prise de parti véhémente en faveur d'intérêts individuels n'est donc rien d'autre que le renforcement dans la durée de l'idéal collectif commun. Si ce dernier est absent, la solidarité organique est affaiblie au point que cette négligence, à long terme, reviendrait à un « véritable suicide moral ${ }^{73}$ ».

En définitive, le rationalisme, l'individualisme et le libéralisme représentent dans la conception de Durkheim l'héritage moral de la tradition sociale européenne, dans laquelle l'exigence d'autonomie morale de l'individu va de pair avec le désir de justice sociale. Cet héritage moral, il ne s'agit pas seulement de le défendre, mais de se battre pour le faire progresser encore. Ce n'est qu'ainsi que naîtra une société véritablement démocratique, dans laquelle l'Etat sera le gardien suprême de l'idéal collectif individualiste, tandis que les organisations professionnelles agiront comme organes de réglementation de l'économie et qu'un processus rationnel d'éducation garantira la formation de personnalités autonomes.

Si l'on y parvient, ce sera finalement la réalisation du programme de la Révolution française, et il apparaitra dans les relations sociales plus d'initiatives personnelles et de perspectives de vie (liberté), de réciprocité (égalité) et de solidarité organique (fraternité ) .

\section{Le sociologue comme individualiste et moraliste}

Partant du diagnostic de crise morale posé par Durkheim, nous avons examiné sa théorie morale sous l'angle de la relation qu'il établit entre ordre social et autonomie personnelle. Dans le cadre de son kantisme sociologique, il propose sur ce point trois arguments. Premièrement, il démontre pourquoi une société fonctionnellement différenciée et pluraliste est structurellement contrainte d'assurer la liberté individuelle dans la coordination des différents droits et devoirs (argument de théorie structurelle). Il examine ensuite les conditions nécessaires à la formation de l'autonomie personnelle. Dans une éducation purement rationnelle, les éléments constitutifs d'une morale individualiste - la conscience du devoir, la désirabilité des idéaux sociaux et la capacité de réflexion - sont transmis grâce à des processus qui suscitent l'esprit de discipline en faveur d'une régularité de conduite et de l'orientation vers un but, qui permettent l'attachement aux groupes sociaux par l'intégration aux divers milieux sociaux (famille, école, profession, nation, humanité) ainsi que par l'intériorisation des différentes règles, et qui favorisent l'autonomie par la 
compréhension rationnelle (argument de théorie de la socialisation). Enfin, une éducation laïque visant à l'autonomie morale ne convainc pas seulement par son orientation rationnelle, mais dans la mesure où elle devient l'objet des sentiments moraux d'une société. Une éthique individualiste ne revêt l'autorité nécessaire que si elle devient un idéal collectif sacré. Dans son examen de l'affaire Dreyfus, Durkheim démontre que le culte de l'individu est effectivement le nouvel idéal collectif sacré et que, pour cette raison, les intérêts de la sûreté de l'Etat ne peuvent jamais être placés, dans une société démocratique, au-dessus des droits de la personnalité d'un individu (argument de sociologie religieuse).

55 Le kantisme sociologique de Durkheim, qui repose sur les trois arguments que nous avons présentés, aboutit néanmoins à une sorte d'«individualisme collectiviste », qui fait problème ${ }^{74}$ pour trois raisons. Relevons en premier lieu son concept équivoque de la société, qui comprend à la fois la réalité sociale et l'idéal moral, et confond donc la dimension empirique et la dimension normative. À la manière de la preuve de l'existence de Dieu de Kant, Durkheim ${ }^{75}$ part de la société considérée comme une instance indépendante pour postuler une morale séculière.

«[...] s'il existe une morale, un système de devoirs et d'obligations, il faut que la société soit une personne morale qualitativement distincte des personnes individuelles qu'elle comprend [...] Nous postulons une société spécifiquement distincte des individus, parce que, autrement, la morale est sans objet, le devoir sans point d'attache. "

Cependant les points d'attache choisis ne sont pas les sociétés existantes, avec leurs inégalités et leurs injustices, mais leur idéal.

«[...] la civilisation, c'est l'ensemble de tous les biens auxquels nous attachons le plus grand prix; c'est l'ensemble des plus hautes valeurs humaines. Parce que la société est à la fois la source et la gardienne de la civilisation, parce qu'elle est le canal par lequel la civilisation parvient jusqu'à nous, elle nous apparaît donc comme une réalité infiniment plus riche, plus haute que la nôtre ${ }^{76}[. .$.$] ».$

La plus forte objection contre cette construction est qu'elle ne semble autoriser aucune solidarité du travail commun au cours duquel les règles de la coopération sont fixées de manière autonome par les individus. Par sa mise en parallèle de la morale archaïque et de la morale moderne, Durkheim reprend à son compte le modèle de relations sociales asymétriques - l'écart de pouvoir entre la conscience collective et l'individu - pour les sociétés modernes, de sorte qu'une conscience collective "sacrée ", même si elle est individualiste, instaure dans tous les cas les règles sociales. Durkheim étaie ce fondement archaïque de la morale moderne par trois arguments qui expliquent la priorité logique de la société face à l'individu : du point de vue chronologique, les sociétés préexistent à l'apparition d'un individu en elles et, en général, elles durent également plus longtemps que l'espérance de vie d'un individu; du point de vue factuel, il existe généralement un corpus de connaissances plus vaste que ce que l'individu peut s'approprier; enfin, du point de vue social, l'influence de la société sur l'individu dépasse nettement les impulsions que ce dernier peut donner en faveur de l'évolution sociale.

De ces arguments pertinents pour la priorité logique de la société, on ne doit cependant pas conclure à sa supériorité morale et condamner ainsi les individus à une attitude de réception passive de la morale. Une telle conclusion serait abusive : des prophètes et des philosophes charismatiques tels que Jésus et Socrate n'ont pas seulement reconnu "les signes du temps » et présenté à la société de leurs époques leurs perspectives d'avenir en matière de morale, ils ont aussi élaboré une doctrine entièrement neuve sur 
ce plan, contrastant nettement avec la structure sociale alors en vigueur. De plus, la priorité logique de la société n'exclut en aucune manière la possibilité de nouvelles formations sociales spontanées se donnant à elles-mêmes leurs propres règles, pas plus qu'elle ne s'oppose à ce que le modèle de la coopération véritable soit progressivement étendu à la société tout entière.

En second lieu, l'équivoque imprègne également la conception de la morale de Durkheim. Conçue à l'origine comme une classification empirique des règles existantes, Durkheim la transforme en une hiérarchie normative contraignante des droits et devoirs sociaux. Le relevé scientifique d'une phénoménologie de la morale ne permet toutefois de tirer des conclusions que sur la portée empirique de règles morales concrètes, pas sur leur validité éthique, si l'on ne veut pas commettre une inadmissible confusion entre être et devoir-être. La conviction de Durkheim selon laquelle une règle est d'autant plus universelle que son domaine d'application est vaste et qu'elle est placée à un haut rang dans l'ordre logique des règles morales l'amène à la conclusion erronée que la règle morale qui a le plus vaste domaine d'application a également la plus grande force d'autorité. Son sophisme consiste à construire l'ordre logique d'un système de philosophie morale en le calquant sur l'ordre sociologique d'un système social, et à penser que la hiérarchie philosophique des droits et des devoirs correspond également à la hiérarchie sociale des règles morales. Du point de vue sociologique, c'est précisément le contraire qui est vrai : plus une règle s'éloigne du rayon d'action immédiat des intérêts individuels, moins son caractère obligatoire est ressenti par l'individu, et moins il va de soi de s'y conformer - il en résulte que le respect de la règle doit être garanti par une instance de sanction développée à cet effet, qui « rappelle » aux individus leurs devoirs et leurs droits en cas de doute.

Troisièmement, dans le cadre de cette conception de la morale, l'autonomie de l' individu se réduit à la compréhension rationnelle de la nécessité sociale des règles morales. L'analogie entre les sciences de la nature et les sciences morales qu'invoque Durkheim sur ce point n'est cependant pas pertinente, puisque les lois naturelles sont infrangibles et immuables, tandis qu'il est non seulement possible d'enfreindre consciemment les règles morales, mais aussi de les transformer par l'initiative individuelle. Le parti-pris unilatéralement sociologique de Durkheim, qui consiste à considérer systématiquement la morale à partir de la perspective de la société, conduit à une sous-estimation tendancielle de la créativité et de la spontanéité individuelles. Cette vision unilatérale résulte du caractère sommaire de sa théorie de la personnalité : s'il décrit le dualisme riche de tensions de la nature humaine, il identifie à tort l'organisme avec l'être individuel et la personnalité avec l'être social, sans prévoir d'instance médiatrice telle, par exemple, que l'identité de soi. Dans cette constellation, la personne humaine se définit dans tous les cas par un primat de la personnalité construite socialement sur les penchants pulsionnels individuels. Deux significations distinctes de la socialisation morale ${ }^{77}$ sont ainsi indûment amalgamées : d'une part la socialisation au sens le plus général, en tant que processus d'« hominisation » qui n'est possible que par l'interaction avec autrui; de l'autre, la transmission de la culture particulière d'une société. Seule la prise en compte de cette distinction permet de concevoir que l'on peut fort bien être né français et avoir grandi en France, et néanmoins, en tant qu'être humain autonome, prendre notablement ses distances visà-vis de la mentalité française. 

comme étant une éthique de l'accommodation anti-individualiste et conservatrice. Durkheim fait lui-même référence aux innovations morales de Socrate et de Jésus, et souligne la nécessité de la rébellion individuelle lorsque la société reste en-deçà de ses possibilités morales. Si ce n'était pas le cas, on voit mal comment il pourrait s'arroger le droit de tendre à sa propre société le miroir de l'anomie et d'exiger la création d'une morale nouvelle.

Si l'on considère pour conclure les trois objections contre sa conception de la société, de la morale et de l'individu, il apparaît que la critique de son individualisme collectiviste porte moins sur sa perspective théorique que sur les conclusions pratiques qu'il en tire. Nous pouvons donc sans difficulté refuser le contenu de ses propositions concrètes en vue d'établir une morale citoyenne, tout en conservant la perspective féconde d'étudier la relation entre structure sociale et conscience morale. Ce n'est qu'en oubliant Durkheim le moraliste que nous pourrons redécouvrir à notre profit Durkheim l'individualiste.

\section{BIBLIOGRAPHIE}

Adorno, T. W. (1976) : « Einleitung », in : Durkheim, E. : (1976) : Soziologie und Philosophie, trad. par E. Moldenhauer, Francfort-sur-le-Main : Suhrkamp, p. 7-44.

Alexander, J. C. (1982) : Theoretical Logic in Sociology. The Antinomies of Classic Thought: Marx and Durkheim, Berkeley/Los Angeles : University of California Press.

Aron, R. (1971) : Hauptströmungen des soziologischen Denkens, vol. 2, trad. F. Becker, Cologne : Kiepenheuer \& Witsch.

Bellah, R. N. (1973) : Introduction à Emile Durkheim On Morality and Society, éd. par R. N. Bellah, Chicago : The University of Chicago Press, p. IX-LV.

Bertram, H. (1980) : « Moralische Sozialisation », in : Handbuch der Sozialisationsforschung, éd. par K. Hurrelmann et D. Ulich, Weinheim / Bâle : Beltz, p. 717-744.

Besnard, P. (éd.) (1983) : The Sociological Domain. The Durkheimians and the Founding of French Sociology, Cambridge / Paris : Cambridge University Press.

Bougie, C. (1930) : «L'œuvre sociologique d'Emile Durkheim. Quelques souvenirs », Europe, 22, p. 281-304 (avec d'autres articles de Davy, Granet, Lenoir, Maublanc).

Clark, T. N. (1968) : « Emile Durkheim and the Institutionalization of Sociology in the French University System », Europäisches Archiv für Soziologie, 9, p. 37-71.

Clark, T. N. (1981) : « Die Durkheim-Schule und die Universität » in : Lepenies, W. (1981b), vol. 2, p. $157-205$.

Davy. G. (1919) : « Emile Durkheim : l'homme », Revue de métaphysique et de morale, 16, p.181-198.

Davy, G. (1920) : « Emile Durkheim : l'œuvre », Revue de métaphysique et de morale, 27, p. 71-112. 
Davy, G. (1960) : « Emile Durkheim », Revue française de sociologie, 1, p. 3-24.

Durkheim, E. (1887a) : « La Philosophie dans les universités allemandes », Revue internationale de l'enseignement, XIII, p. 313-338, 423-440 ; reproduit dans Durkheim (1975c), p. 437-486.

Durkheim, E. (1887b) : «La Science positive de la morale en Allemagne », Revue philosophique, 24, p. 33-58, 113-142, 275-284 ; reproduit dans Durkheim (1975a), p. 267-343.

Durkheim, E. (1888a) : « Cours de science sociale; leçon d'ouverture », Revue internationale de l'enseignement, XV, p. 23-48; reproduit dans Durkheim (1970), p. 77-110.

Durkheim, E. (1888b) : «Introduction à la sociologie de la famille », Annales de la Faculté des Lettres de Bordeaux, 10, p. 257-281; reproduit dans Durkheim (1975c), p. 9-34.

Durkheim, E. (1921) : « La famille conjugale. Conclusion du cours sur la famille », édité par M. Mauss avec un avant-propos, Revue philosophique, 90, p.2-14; reproduit dans Durkheim (1975c), p. 35-49.

Durkheim, E. (1969a [1897]) : Le Suicide, $3^{\mathrm{e}}$ éd., Paris : PUF.

Durkheim, E. (1969b [1950]) : Leçons de sociologie. Physique des mœurs et du droit, $2^{\mathrm{e}}$ éd., Paris : PUF.

Durkheim, E. (1970) : La Science sociale et l'action, édité et préfacé par J. C. Filloux, Paris : PUF.

Durkheim, E. (1974a [1924]) : Sociologie et philosophie, $4^{\mathrm{e}}$ éd., Paris : PUF ; trad. allemande : Soziologie und Philosophie, introduction de T. W. Adorno, trad. par E. Moldenhauer, Francfort-sur-le-Main : Suhrkamp, 1976.

Durkheim, E. (1974b [1925]) : L'Education morale, nouvelle éd., Paris : PUF.

Durkheim, E. (1975) : Textes, 3 vol.,présentés par V. Karady, Paris : Minuit.

- (1975a) : vol. 1, Eléments d'une théorie sociale.

- (1975b) : vol. 2, Religion, morale, anomie.

- (1975c) : vol. 3, Fonctions sociales et institutions.

Durkheim, E. (1978 [1893]) : De la division du travail social, 10e éd., Paris : PUF.

Durkheim, E. (1979 [1912]) : Les Formes élémentaires de la vie religieuse, $6^{\mathrm{e}}$ éd., Paris : PUF.

Durkheim, E. (1980 [1922]) : Education et sociologie, introduction de P. Fauconnet, 4 éd., Paris : PUF.

Durkheim, E. (1981b) : Frühe Schriften zur Begründung der Sozialwissenschaft, édité, préfacé et traduit par L. Heisterberg, Darmstadt / Neuwied : Luchterhand.

Durkheim, E. (1983 [1895]) : Les Règles de la méthode sociologique, $21^{\mathrm{e}}$ éd., Paris : Flammarion.

Fauconnet, P. (1973) : «Das pädagogische Werk Durkheims », Einführung zu E. Durkheim Über Erziehung, Moral und Gesellschaft, Vorlesung an der Sorbonne 1902/1903, Neuwied / Berlin : Luchterhand, p. 7-33.

Filloux, J. C. (1970) : «Introduction », in : Durkheim (1970), p. 5-68.

Freud, S. (1971) : Abriß der Psychoanalyse. Das Unbehagen in der Kultur, $20^{\mathrm{e}}$ éd., Francfort-sur-leMain : Fischer ; trad. fr. : Abrégé de psychanalyse, Paris : PUF, 2001 et Malaise dans la civilisation, Paris : PUF, 1971.

Geiger, R. L. (1981) : « Die Institutionalisierung soziologischer Paradigmen : Drei Beispiele aus der Frühzeit der französischen Soziologie », in : W. Lepenies (1981b), p. 137-156.

Ginsberg, M. (1951) : « Durkheim's Ethical Theory », British Journal of Sociology, 2, p. 210-218. 
Goffman, E. (1975) : Stigma. Über Techniken der Bewältigung beschädigter Identität, Francfort-sur-leMain : Suhrkamp.

Gouldner, A. W. (1958) : «Introduction » zu E. Durkheim Socialism and Saint-Simon, trad. de C. Sattler, Londres : Routledge, p. V-XXVII.

Habermas, J. (1981) : Theorie des kommunikativen Handelns, 2 vol., Francfort-sur-le-Main : Suhrkamp (cit. 1981a et b) ; trad. fr. : Théorie de l'agir communicationnel, 3 vol., Paris : Fayard, 1987. Hofmann, I. (1973) : Bürgerliches Denken. Zur Soziologie Durkheims, Francfort-sur-le-Main : Athenäum.

Kant, E. (1785) : Grundlegung zur Metaphysik der Sitten, in : Kant (1983), p. 1-102 ; trad.fr. : Fondements de la métaphysique des mœurs, Paris : Vrin, 2002.

Kant, E. (1983) : Schriften zur Ethik und Religionsphilosophie, in : Werke, vol. 4, éd. par W. Weischedel, Darmstadt : Wissenschaftliche Buchgesellschaft.

Karady, V. (1981) : « Strategien und Vorgehensweisen der Durkheim-Schule im Bemühen um die Anerkennung der Soziologie », in : Lepenies (1981b), p. 106-262.

König, R. (1976a) : « Emile Durkheim. Der Soziologe als Moralist », in : Käsler, D. (éd.) : Klassiker des soziologischen Denkens, Munich : Beck, vol. 1, p. 312-364.

König, R. (1976b) : « Soziologie der Familie », in : Id. (éd.) : Handbuch der empirischen Sozialforschung, Stuttgart : Thieme, vol. 7 : Familie - Alter, p. 1-217.

König, R. (1978) : Emile Durkheim zur Diskussion. Jenseits von Dogmatismus und Skepsis, Munich / Vienne : Hanser.

Krisam, R. (1972) : « Vorwort » zu E. Durkheim Erziehung und Soziologie, Düsseldorf : Schwann, p. 7-19.

Lepenies, W. (éd.) (1981) : Geschichte der Soziologie. Studien zur kognitiven, sozialen und historischen Identität einer Disziplin, 4 vol., Francfort-sur-le-Main : Suhrkamp (cit. Lepenies 1981a-d).

Lindenberg, S. (1983) : « Zur Kritik an Durkheims Programm für die Soziologie », Zeitschrift für Soziologie, 2, p. 139-151.

Lukes, S. (1973) : Emile Durkheim. His Life and Work. A Historical and Critical Study, Londres : Penguin. Mauss, M. (1971 [1928]) : «Introduction » à E. Durkheim Le Socialisme, Paris : PUF, p. 27-31.

Müller, H.-P. (1983) : Wertkrise und Gesellschaftsreform. Emile Durkheims Schriften zur Politik, Stuttgart : Enke.

Münch, R. (1982) : Theorie des Handelns, Francfort-sur-le-Main : Suhrkamp.

Nisbet, R. A. (1974) : The Sociology of Emile Durkheim, Oxford : Oxford University Press.

Parsons, T. (1968) : The Structure of Social Action. A Study in Social Theory with special reference to a group of recent European writers, New York : The Free Press.

Piaget, J. (1973) : Das moralische Urteil beim Kinde, trad. par L. Goldmann, Francfort-sur-le-Main : Suhrkamp.

Pickering, W. S. F. (éd.) (1979) : Essays on Morals and Education. Introduction, London / Boston / Henley : Routledge \& Kegan Paul.

Pollak, M. (1978) : Gesellschaft und Soziologie in Frankreich. Tradition und Wandel in der neueren französischen Soziologie, Königstein/Ts : Hain. 
Rawls, J. (1979) : Eine Theorie der Gerechtigkeit, trad. par H. Vetter, Francfort-sur-le-Main :

Suhrkamp ; trad. française : Théorie de la justice, Paris : Seuil, 1997.

Rousseau, J.-J. (2012 [1762]) : Du Contrat Social, Paris : Flammarion.

Schriewer, J. (1983) : « Pädagogik - ein deutsches Syndrom? Universitäre Erziehungswissenschaft im deutsch-französischen Vergleich », Zeitschrift für Pädagogik, 29, 3, p. 359-389.

Simmel, G. (1983) : Schriften zur Soziologie. Eine Auswahl, éd. et présenté par H.-J. Dahme et O. Rammstedt, Francfort-sur-le-Main : Suhrkamp.

Tiryakian, E. A. (1978) : « Emile Durkheim », in : Bottomore / Nisbet (éd.) : A History of Sociological Analysis, New York : Basic Books, p. 187-236.

Vogt, W. P. (1982) : «Relativistic absolutism in moral éducation », New York University Education Quarterly, 13 (3), p. 29-33.

Wallwork, E. (1972) : Durkheim, Morality and Milieu, Cambridge, Mass. : Harvard University Press

Wrong, D. (1974) : « Das Theorem der Übersozialisation in der modernen Soziologie », in Mühlfeld, C. / Schmid, M. (éd.) : Soziologische Theorie, Hambourg : Hoffmann und Campe, p. 281-291.

\section{NOTES}

1. Durkheim (1978), p. 404.

2. C'est également ce que pensent Aron (1971), p. 80 sq. ; Bellah (1973), p. IX sq. ; Bertram (1980), p. 717 sq. ; Davy (1919), (1920), p. 71 et (1960) ; Fauconnet (1973), p.11 sq. ; König (1976a), p. 329 sq., (1978) ; Lukes (1973), p. 410 sq. ; Nisbet (1974), p. 187 sq. ; Piaget (1973), p. 369 sq. ; Pickering (1979), p. 3 sq.; Vogt (1982), p. 29 sq.; Wallwork (1972). Ces auteurs proposent également un bon aperçu de la théorie morale et de l'éducation morale chez Durkheim.

3. Au sujet de l'institutionnalisation de la sociologie française sous l'influence de Durkheim, voir Besnard (1983) ; Clark (1968), p. 37 sq., (1981), p. 157 sq. ; Filloux (1970), p. 8 sq. ; Geiger (1981), p. 149 sq. ; Karady (1981), p. 206 sq. ; Müller (1983), p. 61 sq. ; Pollak (1978), p. 7 sq. ; Tiryakian (1978), p. 187 sq.

4. Ce programme commence à être réalisé dès les premiers articles de Durkheim (1887a), (1887b), (1888a), et se développe au fur et à mesure. Voir Durkheim (1983).

5. Durkheim (1978), p. 406.

6. C'est la conscience de cette crise qui le mène à la sociologie et qui induit également l'exigence morale (voire moralisatrice) qu'il adresse à cette discipline. Voir sur ce point Davy (1919) et avant tout Mauss (1971) ainsi que König (1976a), p. 312 sq. et Müller (1983), p. 11 sq.

7. Entre la Révolution française de 1789 et la défaite lors de la guerre franco-allemande de 1870 , suivie de l'instauration de la Troisième République en 1871, la France a connu huit régimes politiques, dont trois monarchies, deux empires et deux républiques, avec en tout quatorze constitutions différentes.

8. La "question sociale » était au centre des débats politiques de l'époque, et elle a également joué un rôle dans la décision de Durkheim de se consacrer à la sociologie. Voir Mauss (1971), p. 27.

9. En France, la question de l'école laïque reste un problème politique irrésolu depuis l'époque des Lumières, puisque les écoles privées, ou écoles libres, sont encore aujourd'hui entre les mains de l'Église catholique et que les tentatives de supprimer les subventions de l'État pour ce type d'établissements suscitent toujours des protestations massives.

10. Durkheim (1970), p. 109. 
11. Cet usage synonyme des termes s'explique par le fait qu'au XIX ${ }^{\mathrm{e}}$ siècle, on avait établi, sous le nom de statistiques morales, des listes systématiques d'informations concernant les naissances, les mariages, les crimes, les suicides et les décès, qui furent utilisées par les premiers sociologues empiriques - d'Adolphe Quetelet jusqu'à Frédéric Le Play, un contemporain de Durkheim comme bases de données pour leurs tentatives d'expliquer la vie sociale d'un point de vue sociologique.

12. Voir par exemple la surprise que provoque le fort engagement moral de Durkheim chez Célestin Bouglé (1930), p. 283.

13. À titre d'exemple de ces analyses, voir Adorno (1976) et Hofmann (1973). Lindenberg ([1983], p. 139) affirme que « le programme de Durkheim a représenté et représente toujours un obstacle au développement de la sociologie ». L'influence féconde du programme de Durkheim pour une macrosociologie structurelle empirique plaide cependant contre la thèse de Lindenberg. Le succès d'un paradigme se mesure-t-il à autre chose qu'à la fécondité heuristique de son programme?

14. Nous sommes contraints de renoncer ici à examiner la question des influences auxquelles sa pensée a été soumise, la complexe problématique de l'évolution de son œuvre ainsi que le statut de sa théorie morale dans l'éventail des sciences sociales de l'époque.

15. Ainsi que le pensent des auteurs récents comme Alexander (1982), p. 119 sq. ; Habermas (1981b), p. 86 sq. ; et Münch (1982), p. 281 sq.

16. Voir Durkheim (1887a) et (1887b).

17. Durkheim (1887a), p. 330 ou (1975c), p. 456.

18. Le projet de Durkheim peut donc être considéré comme le pendant sociologique de l'œuvre philosophique de Kant, les Fondements de la métaphysique des mœurs et du droit de 1785 (Kant [1983]). Cependant, Durkheim parle aussi de "physiologie du droit et des mœurs", ce qui rappelle plutôt la sociologie de Comte. Dans ce contexte, voir la présentation générale des cours de sociologie de la morale à Bordeaux et à Paris chez Lukes (1973), p. 617 sq.

19. Voir Wallwork (1972), p. 5 sq. et Müller (1983), p. 22 sq.

20. Durkheim (1978), p. XXXVII.

21. Durkheim (1983), p. 120.

22. Durkheim (1887b), p. 42 ou (1975a), p. 278.

23. Durkheim (1974b), p. 74.

24. Durkheim (1887b), p. 56 ou (1975a), p. 295.

25. Durkheim (1978), p. XXXVII.

26. Le texte Détermination du fait moral est reproduit, accompagné d'extraits de la discussion, dans le volume Sociologie et philosophie (Durkheim [1974a]), dont il forme le deuxième chapitre.

27. Voir Durkheim (1974b).

28. Durkheim (1974a), p. 51.

29. Durkheim (1974b), p. 21.

30. Ce sont les exemples que Durkheim (1983) donne lui-même comme des manifestations de la contrainte sociale, avant d'introduire la distinction entre règles techniques et règles morales.

31. Durkheim (1974a), p. 59 sq.

32. Durkheim (1974a), p. 60.

33. Durkheim (1974a), p. 52.

34. Durkheim (1974a), p. 62.

35. Durkheim (1974b), p. 82.

36. Durkheim (1974b), p. 51.

37. Durkheim (1978), p. 394.

38. Le traité de Durkheim De la division du travail social, publié en 1893 et qui a pour origine son travail de thèse, portait à l'origine le sous-titre Étude sur l'organisation des sociétés supérieures, ce qui montre que l'auteur s'intéresse au premier chef à la société moderne. 
39. Durkheim (1978), p. 201.

40. Durkheim (1978), p. 46.

41. Durkheim (1978), p. 143.

42. Durkheim (1980), p. 74.

43. Durkheim (1978), p. 74

44. Durkheim (1978), p. 99 sq.

45. Durkheim (1978), p. 206.

46. Durkheim (1978), p. 6.

47. L'Éducation morale (Durkheim [1974b]) reprend le texte des leçons données régulièrement par Durkheim à Bordeaux entre 1889 et 1902, puis à Paris à partir de 1902, et qui contiennent les éléments fondamentaux pour édifier un système d'éducation laïque. Voir aussi à ce sujet Schriewer (1983), p. 74.

48. Durkheim (1974b), p. 3.

49. Durkheim (1974b), p. 41.

50. Durkheim (1974b), p. 39 sq.

51. Durkheim (1969a).

52. Durkheim (1969a), p. 43.

53. Durkheim (1969a), p. 42.

54. Voir Durkheim (1888b) et (1921), ainsi que König (1976b), p. 60 sq.

55. Voir la deuxième préface de Durkheim (1978), p. I sq., et Durkheim (1969b), p. 41 sq. Voir aussi Müller (1983), p. 146 sq.

56. Durkheim (1974b), p. 64.

57. Durkheim (1974b), p. 98.

58. Durkheim (1974b), p. 97.

59. Durkheim (1974b), p. 100.

60. Voir Durkheim (1979), p. 314 sq

61. Durkheim (1974a), p. 76.

62. Durkheim (1974a), p. 100.

63. Durkheim (1974b), p. 101.

64. Piaget (1973), p. 385 sq.

65. Freud (1971).

66. Goffman (1975).

67. Georg Simmel ([1983], p. 267 sq.) attirait déjà l'attention sur ce point en 1917, puisqu'il distinguait entre l'individualisme germanique, qui vise, en dépit de toutes les contraintes extérieures, le caractère unique de l'individu, et l'individualisme latin orienté vers un type général.

68. Rousseau (1762).

69. Durkheim (1970), p. 264 sq.

70. Dans le sens de Rawls (1979), p. 493 sq.

71. Durkheim (1970), p. 264 sq.

72. Ibid.

73. Durkheim (1970), p. 274.

74. Sur ce point, voir Aron (1971), p. 85 sq. ; Bellah (1973), p. IX ; Ginsberg (1951), p. 210 sq. ; Gouldner (1958), p. XXI ; Lukes (1973), p. 296 sq., p. 497 sq.; Müller (1983), p. 88 sq.; Parsons (1968), p. 376 sq. ; Piaget (1973), p. 391.

75. Durkheim (1974a), p. 70.

76. Durkheim (1974a), p. 73 sq.

77. Wrong (1974), p. 290. 
INDEX

Mots-clés : morale, individualisme, société, culte de l'individu, physique des mœurs et du droit Schlüsselwörter : Moral, Individualismus, Gesellschaft, Kult des Individuums, Physik der Sitten und des Rechts

\section{AUTEURS}

\section{HANS-PETER MÜLLER}

Hans-Peter Müller est professeur de sociologie à l'Université Humboldt de Berlin. Pour plus d'informations, voir la notice suivante. 